\title{
LETTERS
}

\section{Response to Capsule Commentary on Merlin et al., Managing Concerning Behaviors in Patients Prescribed Opioids for Chronic Pain: A Delphi Study}

\author{
Jessica S. Merlin, MD, PhD, MBA ${ }^{7,2}$, Jane M. Liebschutz, MD, MPH', and Joanna L. Starrels, MD, $M S^{3}$ on \\ behalf of the Collaboration and Resources for Pain and Opioid Opinion Leaders (CARPOOL) \\ investigator team
}

'Center for Research on Healthcare, Division of General Internal Medicine, University of Pittsburgh School of Medicine, Pittsburgh, PA, USA; ${ }^{2}$ Division of Infectious Diseases, University of Pittsburgh School of Medicine, Pittsburgh, PA, USA; ${ }^{3}$ Division of General Internal Medicine, Albert Einstein College of Medicine and Montefiore Medical Center, Bronx, NY, USA.

J Gen Intern Med 33(6):783

DOI: $10.1007 / \mathrm{s} 11606-018-4402-1$

(c) Society of General Internal Medicine 2018

$\mathrm{D}$ r. Laws' commentary on our manuscript "Managing Concerning Behaviors in Patients Prescribed Opioids for Chronic Pain: A Delphi Study"1 misrepresents and misinterprets both our study and the CDC Guideline for Prescribing Opioids for Chronic Pain. ${ }^{2}$

The CDC Guideline recommendation about tapering opioids is based on the overall balance of risks vs. benefits. For some behaviors or events, the risk is so high that one occurrence tips the balance (e.g., a nonfatal overdose event). However, a much more common scenario is a behavior such as missed visits or running out of medications early; in primary care, cohorts up to $78 \%$ of patients on opioids have at least one such concerning behavior during treatment. ${ }^{3}$ Such behaviors do not always represent a serious risk that immediately tips the balance. How to respond to behaviors that do not immediately tip the balance is not well addressed in the CDC Guideline due to a lack of empirical evidence. This is precisely why we turned to a rigorous Delphi process to harness expert clinicians' opinions.

Notably, the Delphi participants rarely regarded tapering as an important approach to the first occurrence of behaviors addressed in the study. Rather, initial steps such as reviewing the opioid treatment agreement, providing non-opioid pain treatments, and closer monitoring were favored. Experts endorsed tapering generally after more information was gathered, for example, when a pattern of behavior emerged; in our opinion, this represents the tipping of the risk vs. benefit balance and is consistent with the CDC Guideline.

Laws questions our participants' qualifications. We systematically recruited from professional groups enriched with chronic pain management and opioid prescribing experts. Eligible participants were clinicians who self-reported that

Published online March 19, 2018 opioids for chronic pain was an area of expertise; for example, they "have taught others, published, or are considered a resource for other clinicians on this topic." 4

The commentary also questions the rigor of the Delphi approach. Our findings were based on expert providers' collective experiences, not "intuitions." The Delphi method is a well-regarded, scientifically accepted consensus approach and represents a level of evidence. ${ }^{5}$

We agree that our findings should not replace clinical judgement and need to be rigorously tested in primary care settings. The findings provide a framework for future research and for aiding front-line clinicians in navigating the clinical uncertainty we face when operationalizing current guidelines in patient care.

Financial Support Information: Dr. Merlin received support from the National Institute of Mental Health (K23MH104073[JSM]).

Corresponding Author: Jessica S. Merlin, $M D, P h D, M B A$; Center for Research on Healthcare, Division of General Internal MedicineUniversity of Pittsburgh School of Medicine, Pittsburgh, PA, USA (e-mail: merlinjs@pitt.edu).

\section{REFERENCES}

1. Merlin JS, Young SR, Starrels JL, Azari S, Edelman EJ, Pomeranz J, et al. Managing Concerning Behaviors in Patients Prescribed Opioids for Chronic Pain: A Delphi Study. J Gen Intern Med. 2017. doi: https://doi. org/10.1007/s11606-017-4211-y

2. Dowell D, Haegerich TM, Chou R. CDC Guideline for Prescribing Opioids for Chronic Pain - United States, 2016. MMWR Recomm Rep. 2016;65(1):1-49.

3. Vowles KE, McEntee ML, Julnes PS, Frohe T, Ney JP, van der Goes DN. Rates of opioid misuse, abuse, and addiction in chronic pain: a systematic review and data synthesis. Pain. 2015;156(4):569-76.

4. Merlin JS, Young SR, Azari S, Becker WC, Liebschutz JM, Pomeranz J, et al. Management of problematic behaviours among individuals on longterm opioid therapy: protocol for a Delphi study. BMJ Open. 2016;6(5):e011619.

5. Campbell SM, Braspenning J, Hutchinson A, Marshall M. Research methods used in developing and applying quality indicators in primary care. Qual Saf Health Care. 2002;11(4):358-64. 